\title{
Super-Antigens and Human Pathology: Always an Interesting Topic
}

\section{Sur $\mathbf{G}^{1}$, Sporis $\mathrm{D}^{1}$, Kudor-Szabadi $\mathrm{L}^{1}$ and Samasca $\mathbf{G}^{2 *}$}

${ }^{1}$ Departments of Pediatrics II, University of Medicine and Pharmacy luliu-Hatieganu, Cuj-Napoca, Romania

${ }^{2}$ Departments of Immunology, University of Medicine and Pharmacy luliu-Hatieganu, Cuj-Napoca, Romania

\begin{abstract}
Superantigens represent a group of proteins that are able to activate a large number of different $\mathrm{T}$ lymphocytes clones. Superantigens are potent activators of $\mathrm{CD}^{+} \mathrm{T}$ cells, causing rapid and massive proliferation of cells and cytokine production. Superantigen administration is able to significantly enhance ineffective anti-tumor immune responses, resulting in potent and long-lived protective anti-tumor immunity. Superantigens are more well-known for the role they play in diseases. Some studies argue that in multiple sclerosis superantigens can induce severe relapses and activate auto-reactive T cells not involved in the initial bout of disease. Superantigens are produced by pathogenic microbes (bacteria, viruses and mycoplasma) into the cells and then are released outside the cells as mature toxins. There are two types of super-antigens: endogenous (viral) and exogenous (bacterial). They escape intracellular processing by binding directly to $\mathrm{MHC} \mathrm{II}$, outside the conventional site where antigens bind. Interaction of super antigens with $\mathrm{T}$ lymphocyte can produce the inhibition of the cellular response to energy. Superantigens may be involved in T cell apoptosis. Best known super-antigens are: staphylococcus aureus enterotoxins $A$ and $B$ and the streptococcus exotoxins $A-G$, streptococcus wall $M$ protein, exotoxins produced by Clostridium perfringens, Yersinia enterocolitica. Recently it was ascertained that super-antigens play an important role in human pathology, with a dramatic effect on the immune system. These molecules are involved in various diseases, such as: Toxic shock syndrome, Kawasaki disease, Eczema, Guttate psoriasis, Rheumatoid arthtritis, Diabetes mellitus, Scarlet fever, Nasal polyps.
\end{abstract}

Keywords: Children; Diseases; Super antigens

\section{Introduction}

Staphylococcal enterotoxin effects have been observed since 1960, but only in 1990 Kappler and Marrack named it super-antigen [1]. Super-antigens consist of a group of bacterial and viral proteins that are able to activate a large number of different and various clones of $\mathrm{T}$ lymphocytes $[1,2]$. $\mathrm{T}$ cell activation by the super antigen is different from activation by conventional antigens. The conventional activation antigen is necessary to be taken by an antigen presenting cell (APC). For the antigen to be recognized the presence of the major histocompatibility complex (MHC) is mandatory. MHC is found in two types: class I, found on all cell types that are loaded with peptides from their proteins. This type is fixed on the cells that will activate CD8 lymphocytes. Class II is present only in APC such as monocytes, macrophages and dendritic cells. This molecule sets peptides derived from foreign proteins, which were previously processed by APC, CD 4 lymphocyte receptors $[2,3]$.

\section{Ways of activation of T lymphocytes by super antigen}

Super-antigens escape intracellular processing by binding directly to MHC II, outside the conventional site where antigens bind. Super antigens then bind directly MHC II with specific receptors of $\mathrm{T}$ lymphocytes (TCR), which are members of the VB family. In this way it occurs a polyclonal activation of $\mathrm{T}$ lymphocytes with massive release of cytokines involved in inflammatory processes associated with different diseases. Activation of TNF and IL I cause vascular endothelial changes in TSS (Toxic Shock Syndrome). Activation of IL 6 and GM-CSF produces pro-inflammatory and pro-thrombotic effects in Kawasaki disease. Interaction of super antigens with $\mathrm{T}$ lymphocyte can produce the inhibition of the cellular response to energy. Super-antigens may be involved in $\mathrm{T}$ cell apoptosis which can be prevented by interferon administration $[3,4]$.

Mainly there are known two types of super-antigens: endogenous (viral) and exogenous (bacterial). Bacterial super-antigens are represented, in fact, by the toxins released by bacteria. Best known super-antigens are: staphylococcus aureus enterotoxins $A$ and $B$ and the streptococcus exotoxins A-G, wall streptococcus M protein, exotoxins produced by Clostridium perfringens, Yersinia enterocolitica. Recently, it was described a protein released by Mycoplasma arthritidis, having the role of a super-antigen $[5,6]$.

In the past few years there has been an explosion of information regarding viral super antigens, their infectivity and viral replication. Animal experiments were performed to elucidate the dramatic and variable effect of super antigens on Tlymphocytes and on other immune processes. In the end it was clearly certified the role of super-antigens in human pathology. Remember that in these diseases super antigens have a proven effect: toxic shock syndrome, Kawasaki disease and other autoimmune diseases, worsening secondary immunodeficiency in HIV and a number of dermatological diseases [5,6]. Still, there are many questions about the super-antigens: it remains unclear how, despite having different structure may interact with MHC II and then with the same region of the TCR, causing $\mathrm{T}$ cell activation through a common pathway. Functional role of bacterial antigens also remains to be explained. The infections with major consequences by producing toxins are still rare events. One hypothesis trying to explain the action of these super-antigens which differs substantially in terms of structure, show that stimulation of $\mathrm{T}$ cells produces the release of inflammatory

*Corresponding author: Samasca Gabriel, Departments of Pediatrics II University of Medicine and Pharmacy luliu-Hatieganu, Cuj-Napoca, Romania, E-mail: Gabriel.Samasca@umfcluj.ro

Received March 22, 2013; Accepted April 20, 2013; Published April 27, 2013

Citation: Sur G, Sporis D, Kudor-Szabadi L, Samasca G (2013) Super-Antigens and Human Pathology: Always an Interesting Topic. J Bioequiv Availab 5: 125-128. doi:10.4172/jbb.1000146

Copyright: (c) 2013 Sur G, et al. This is an open-access article distributed under the terms of the Creative Commons Attribution License, which permits unrestricted use, distribution, and reproduction in any medium, provided the original author and source are credited. 
cytokines, but mediates the suppression of the immune response of the host. It seems that only the top of the iceberg is seen regarding the role of the super-antigens in the human pathology. There have been released new therapeutic strategies on the mechanisms of diseases caused by super-antigens. Finally we can say that these super-antigens have a dramatic effect on the immune system. Now, the question is whether these molecules or their changes can be used as specific modulators of the immune system to treat the diseases. There were isolated antigen specific receptors of T cell-MTCR that recognize short peptide antigens of T lymphocytes, derived from intracellular proteins, such as tumor associated antigens or antigen peptides viruses. These are presented on the cell surface by HLA molecules and are targets for monoclonal mTCR. These receptors with high affinity can be used to treat tumors or can target against cytokines [3].

\section{Diseases caused by viral super-antigens}

Gloves and socks syndrome: Gloves and socks syndrome was described in 1996 by Harris. This syndrome is characterized by symmetric edema and erythema of the hands and feet, giving the appearance of gloves and socks. The erythema can become papular and purpuric. It is accompanied by itching and sometimes by pain. There is a clear separation line on the edge of erythema on the hands and feet, giving the appearance of gloves and socks. Interesting to note in this syndrome is the occurrence of polymorphic lesions in the mouth [7].

In some cases systemic symptoms as fatigue, arthralgia, headache and lymphadenopathy may occur. Fever may appear within 3-4 days of palmoplantar erythema. The disease usually has a self-limited evolution, but in some cases intravenous gamma globulin is indicated. This disease, especially if it is accompanied by polymorphous lesions of the mouth may be very difficult to differentiate from Kawasaki disease. Super-antigens viral parvovirus B19 and B6 Coxackie were incriminated in causing the disease. We recently had a case of glovessocks syndrome and immunological determinations certified the involvement of parvovirus B19 [8].

The pathogenesis of this syndrome is still not completely understood. Parvovirus B19 is a member of the erythrovirus family. A receptor for Parvovirus B19 is present on bone marrow erythroid precursor cells, endothelial cells of dermal vessels, and keratinocytes. An immunohistochemical study with specific anti-B19V antibodies showed the presence of viral antigens on both epidermal and endothelial cells. Parvovirus B19 is multiplied in cells with rapidly dividing such as red cell precursors. Occasionally, it may infect leukocytes, especially neutrophils. Parvovirus B19 proteins have a cytotoxic effect on megakaryocytes. A small number of reports in the literature show that parvovirus B19 can infect other cells, causing encephalitis, myocarditis, hepatitis. Seropositivity rate in the U.S. and worldwide is $5-10 \%$ in children between 2 and 5 years, $50 \%$ at 15 years, $60 \%$ at 30 years and $90 \%$ over 60 years $[7,8]$.

$\mathrm{B} 19 \mathrm{v}$ equally affects both genders. Clinically B19v may produce purpuric rush (gloves and socks), aplastic crisis in patient with severe anemia.

Rarely, B19v can cause myocarditis, encephalitis, vasculitis, glomerulonephritis. It has been reported that B19v infection was associated with idiopathic thrombocytopenic purpura, HenochSchoenlein, pseudo appendicitis. Pregnant women, if they are infected, fetal hydrops may occur (more often if infection occurs before 20 weeks of gestation). It is also known that $20 \%$ of infections with $\mathrm{B} 19 \mathrm{v}$ may be asymptomatic. Generally B19V infection cannot be distinguished from other viral infection if the exanthema is missing. Often children complain of fever without malaise. Splenomegaly may be present. Children with aplastic crisis never have rushed. Articular manifestations of the hands, elbows and knees may occur. It is currently recognized that "the fifth disease" with cutaneous manifestations, a plastic crises gloves and socks syndrome are caused exclusively by B19v. The virus is spread worldwide and infects only humans. It may be transferred vertically from mother to child through saliva droplets, transfusion and percutaneous exposure to blood. B19v can be transmitted through blood products, such as immunoglobulin, packed red blood cells or platelet and clotting factors. Because it does not have the tire it is very resistant to heat, cold and organic solvents. It can be revealed by determining immunoglobulin $\mathrm{M}$ by ELISA method. In patients with aplastic crisis who are infected with $\mathrm{B} 19 \mathrm{v}$, reticulocytes are $0-1 \%$ and hemoglobin decreases by at least $2 \mathrm{~g} / \mathrm{dl}$ from the previous level $[7,8]$.

Toxic shock syndrome staphylococcal and streptococcal: Toxic shock syndrome (TSS) is a toxin mediated disease, an inflammatory response characterized by high fever, hypotension, diffuse erythematous rash, and multiple organ dysfunctions, which may rapidly progress to severe and intractable shock. TSS is caused by staphylococcal or streptococcal exotoxins. It was first described by Todd in 1978 in 7 children aged between 8 and 17 years who had staphylococcus aureus infections. In 1981 the U.S. there was an epidemic of TSS in women using superabsorbent tampons during menstruation. TSS is related to many other bacterial infections such as pneumonia, osteomyelitis, sinusitis, skin and gynecological infections [9].

TSS is caused by the nonspecific stimulation of T lymphocytes by superantigens. Toxins produced by the bacteria Staphylococcus aureus and Streptococcus pyogenes are superantigens involved in TSS. Infection with $S$. aureus produces classical TSS, whereas $S$. pyogenes causes a modified form of TSS known as either streptococcal TSS. Superantigen binding of T cell receptors and MHC class IIs on antigen presenting cells activates the $\mathrm{T}$ lymphocyte and release in circulation pro inflammatory factors. In this process each body system may be involved, including cardiovascular, renal, hepatic, hematologic, CNS, musculoskeletal, mucous membranes. The incidence of staphylococcal TSS is 2.4 to 16 per 100,000 and of the streptococcal TSS is $10-20$ per 100,000 in the U.S. Mortality is $3 \%$ in the staphylococcal TSS, in the streptococcal is $30-70 \%$ and in recurrent cases is between $30-40 \%[9,10]$.

Clinical manifestations: the symptoms are similar to a point in staphylococcal and streptococcal toxic shock, the difference is that in streptococcal toxic shock clinical manifestations are more brutal. There is a prodrome of 2-3 days and then symptoms as fever, hypotension, organ failure (liver, kidney, respiratory) install and finally, disseminated intravascular coagulation. In streptococcal TSS there is a rash sometimes scarlet-fever-like desquamation or soft tissue necrosis, fasciitis, myositis and gangrene $[9,11]$.

Clinical examination findings: high fever, hypotension, diffuse rash, events with occasional patches of skin and bruising and peeling after 1-2 weeks. Rash initially appears on the trunk, then it extends on hands and feet, on the palms and plants. Signs of multiple organs failure are: hepatic and renal failure, ventricular arrhythmia. It can also detect signs of disseminated intravascular coagulation, acute respiratory distress, necrotizing fasciitis and myositis, altered consciousness, mucosal inflammation, conjunctivitis, vaginitis, pharyngitis.

Center for Disease Control and Prevention (CDC) has proposed 
the following criteria for diagnosis of staphylococcal TSS:

-Fever, hypotension and rash

-Failure of 3 organs or systems

-Serological exclusion of leptospirosis, measles, hepatitis B, autoimmune diseases and venereal diseases with positive VDRL

CDC criteria for diagnosis of streptococcal TSS:

-Isolation of group A streptococcus at different levels: blood, CSF, surgical wounds, throat

-Hypotension that did not exist previously

-Failure of two or more organs or systems [10-12].

Note that in the absence of protective immunity TSS may return. The risk of TSS recurrence may be due to superabsorbent tampons, postoperative wounds, postpartum TSS, nasal swabs, bacterial infections, viral infections (influenza, chickenpox), diabetes, chronic heart disease or chronic lung disease.

The prognosis is generally unfavorable for streptococcal TSS, where the mortality rate can reach $70 \%$ and the recurrence rate is $40-50 \%$.

Prehospital treatment involves fluid administration to avoid hypotension and oxygen. In hospital fluid administration continues to control blood pressure, oxygen, cardiac and respiratory monitoring. It should be given intravenous gamma globulin to neutralize toxins. Surgical consultation is necessary to decide whether it needs drainage, fasciotomy or elimination of infected tissue. Patients will be hospitalized into an intensive care unit, will be monitored for hemodynamic, respiratory and hear parameters. Antibiotics will be administered and dialysis for kidney failure will be applied to those who need it [11-13].

Kawasaki disease: It was first described by Tomisaku Kawasaki in 1967. Kawasaki disease raises many questions regarding etiology. It is a generalized vascular disease with unknown etiology with different names: mucocutaneous lymph node syndrome, infantile periarteritis. Current evidence suggests that there is an initial infectious trigger consistent with the presence of superantigenic activity. Several investigators have shown selective expansion of T cell receptor (TCR) $\mathrm{V} \beta 2$-bearing $\mathrm{T}$ cells in peripheral blood during the acute phase of $\mathrm{KD}$. Vasculitis is severe in medium caliber arteries, and can affect the arterioles, capillaries, veins and sometimes larger arteries $[14,15]$.

Major clinical signs of Kawasaki disease are:

1. Fever usually higher than $39.4^{\circ} \mathrm{C}$ for 5 -day, high or remittent character, may last 1-2 weeks without treatment, does not respond to antipyretics and resolves within 1-2 days after administration of iv gamma globulins

2. Bilateral nonexudative conjunctivitis

3. Oropharyngeal lesions: erythema of the mucous membranes, congestive cheilitis, raspberry tongue, without Koplik sign and without ulceration

4. Changes of the extremities: erythema and endured palmar and plantar edema, nail scaling which appears 2 weeks after onset

5. Erythematous rash: diffuse maculopapular rash, scarlet-feverlikerush, multiforme erythema, fine pustules on the extensor surfaces without blisters or bubbles note
6. Cervical lymphadenitis: Diameters approximately $1.5 \mathrm{~cm}$, present in $50-75 \%$ of patients, and do not respond to antibiotic treatment. It is required exclusion of the other conditions that generate lymphadenopathy $[16,17]$.

Important biological signs: accelerated ESR, PCR greatly increased. Phenotypic $\mathrm{T}$ lymphocytes in the acute phase show a polyclonal expansion of $\mathrm{T}$ lymphocytes corresponding variables Vbeta3 family and vBET 8 . This can prove that Kawasaki disease is caused by staphylococcal enterotoxin superantigens and streptococcal exotoxins. Various determinations from different pathological products can highlight the two bacteria [14].

The goal of treatment is to prevent coronary arteritis. High doses of IV gamma globulin, $2 \mathrm{~g} / \mathrm{kg}$ are indicated. It is important to be administered within 10 days or closer to the onset of disease. Also, aspirin reduces inflammatory reactions and maintain hematological parameters $[14,15]$.

Acral erythema-desquamation syndrome relapsed: It is most common in adolescents and adults, manifested by a sudden rash, wellshaped, sometimes with edema in the anal region and then extends to both buttocks and proximal thighs. In some cases edematous erythema can be seen at the hands and feet, lips and nostrils. The pharinx is congestive and the tongue is raspberry. In some cases may occur cervical lymphadenopathy. In acral erythema-desquamation syndrome relapsed implication of super-antigens is possible. There have been described cases of streptococcal exotoxins with super-antigen role.

Manifestation of the disease occurs after a staphylococcal or streptococcal angina, with the advent of an erythema-edema which can became acral. In the end desquamation may be seen. Given the very pronounced similarity with Kawasaki disease, some authors consider this syndrome as a subtle form of Kawasaki disease [18].

Guttate psoriasis: It is characterized by the appearance of erythema on the buttocks, trunk and arms and sometimes on the legs. These lesions occur in a patient with latent chronic psoriasis.

Many studies have shown that in the pathogenesis of psoriasis T cells play an important role, but it is not known precisely what factors are responsible for the activation of these lymphocytes. In these patients have found that an outbreak of streptococcal throat can lead to development of signs of guttate psoriasis. Streptococcal exotoxins manage to activate lymphocytes by a binding mechanism in the region vBET, with all the consequences. There were obtained good results in patients with latent psoriasis who experienced episodes of guttate psoriasis by administration of moldamin, demonstrating involvement of streptococcal superantigens in this disease [19].

\section{References}

1. Alouf JE, Muller-Alouf $\mathrm{H}$ (2003) Staphylococcal and streptococca superantigens: molecular, biological and clinical aspects. Int J Med Microbiol 292: 429-440.

2. DeVries AS, Lesher L, Schlievert PM, Rogers T, Villaume LG, et al. (2011) Staphylococcal toxic shock syndrome 2000-2006: epidemiology, clinical features, and molecular characteristics. PLoS One 6: e22997.

3. Watson AR, Lee WT (2006) Defective T cell receptor-mediated signa transduction in memory CD4 T lymphocytes exposed to superantigen or anti-T cell receptor antibodies. Cell Immunol 242: 80-90.

4. Lappin E, Ferguson AJ (2009) Gram-positive toxic shock syndromes. Lancet Infect Dis 9: 281-290.

5. Sriskandan S, Faulkner L, Hopkins P (2007) Streptococcus pyogenes: Insight into the function of the streptococcal superantigens. Int J Biochem Cell Bio 
Citation: Sur G, Sporis D, Kudor-Szabadi L, Samasca G (2013) Super-Antigens and Human Pathology: Always an Interesting Topic. J Bioequiv Availab 5: 125-128. doi:10.4172/jbb.1000146

39: $12-19$.

6. Brouillard JN, Gunther S, Varma AK, Gryski I, Herfst CA, et al. (2007) Crystal structure of the streptococcal superantigen Spel and functional role of a nove loop domain in $\mathrm{T}$ cell activation by group $\mathrm{V}$ superantigens. J Mol Biol 367: 925-934

7. Bilenchi R, De Paola M, Poggiali S, Acciai S, Feci L, et al. (2012) Papularpurpuric "gloves and socks" syndrome. G Ital Dermatol Venereol 147: 119-121.

8. Heckler GT, Dal Ri NM, Almeida JÃnior HL (2012) Case for diagnosis Papular-purpuric gloves and socks syndrome. An Bras Dermatol 87: 793-794.

9. Ford N, Hargreaves S, Shanks L (2012) Mortality after fluid bolus in children with shock due to sepsis or severe infection: a systematic review and metaanalysis. PLoS One 7: e43953.

10. Ikebe T, Ato M, Matsumura T, Hasegawa H, Sata T, et al. (2010) Highly frequent mutations in negative regulators of multiple virulence genes in group $A$ streptococcal toxic shock syndrome isolates. PLoS Pathog 6: e1000832.

11. Li J, Wang W, Xu SX, Magarvey NA, McCormick JK (2011) Lactobacillus reuteri-produced cyclic dipeptides quench agr-mediated expression of toxic shock syndrome toxin-1 in staphylococci. Proc Natl Acad Sci U S A 108: 33603365 .
12. Shime N, Kawasaki T, Saito O, Akamine Y, Toda Y, et al. (2012) Incidence and risk factors for mortality in paediatric severe sepsis: results from the national paediatric intensive care registry in Japan. Intensive Care Med 38: 1191-1197.

13. Venkataraman, Ramesh (2010) Toxic Shock Syndrome.

14. Kuo HC, Yang KD, Chang WC, Ger LP, Hsieh KS (2012) Kawasaki disease: an update on diagnosis and treatment. Pediatr Neonatol 53: 4-11.

15. Rowley AH, Shulman ST (2010) Pathogenesis and management of Kawasak disease. Expert Rev Anti Infect Ther 8: 197-203.

16. Choi JY, Park SY, Choi KH, Park YH, Lee YH (2013) Clinical characteristics of Kawasaki disease with sterile pyuria. Korean J Pediatr 56: 13-18.

17. Manlhiot C, Christie E, McCrindle BW, Rosenberg H, Chahal N, et al. (2012) Complete and incomplete Kawasaki disease: two sides of the same coin. Eur J Pediatr 171: 657-662

18. Manders SM, Heymann WR, Atillasoy E, Kleeman J, Schlievert PM (1996) Recurrent toxin-mediated perineal erythema. Arch Dermatol 132: 57-60.

19. Aydogan K, Tore O, Akcaglar S, Oral B, Ener B, et al. (2013) Effects of Malassezia yeasts on serum Th1 and Th2 cytokines in patients with guttate psoriasis. Int J Dermatol 52: 46-52. 